\title{
Influência da Classe Diamétrica nos Índices de Rachadura da Madeira em Liquidambar styraciflua
}

\author{
Israel Luiz de Lima ${ }^{1}$, Jaime Anísio de Freitas ${ }^{1}$, José Nivaldo Garcia ${ }^{2}$ \\ ${ }^{1}$ Instituto Florestal do Estado de São Paulo, São Paulo/SP, Brasil \\ ${ }^{2}$ Departamento de Ciências Florestais, Escola Superior de Agricultura "Luiz de Queiroz" - ESALQ, \\ Universidade de São Paulo - USP, Piracicaba/SP, Brasil
}

\begin{abstract}
RESUMO
As tensões de crescimento das árvores podem ser avaliadas por meio da análise das rachaduras de extremidade observadas em pranchas verdes e secas. Dessa forma, este trabalho teve como objetivo estudar a evolução do comprimento das rachaduras, durante o processo de secagem natural da madeira serrada de Liquidambar styraciflua, de 24 anos, em função de diferentes classes de diâmetro (superior, mediana e inferior) e duas posições verticais, ao longo da altura da árvore. De acordo com os resultados obtidos, pôde-se concluir que as rachaduras de extremidade de prancha seca ou verde foram influenciadas apenas pela classe de diâmetro, em que a classe superior apresentou os maiores comprimentos de rachaduras e a classe inferior, os menores. Foi obtida uma relação positiva entre rachadura de prancha seca e rachadura de prancha verde.
\end{abstract}

Palavras-chave: madeira serrada, tensão de crescimento, rachadura.

\section{Influence of Diameter Classes on the End Split Rates of Liquidambar styraciflua Sawn Wood}

\begin{abstract}
The growth stresses of trees can be evaluated by means of the analysis of the end splitting on green and dry boards. Thus, this work aimed to study the evolution of the splitting length during the process of natural drying in the sawn wood of 24-year-old Liquidambar styraciflua, as a function of different diameter classes (upper, middle and lower) and two vertical positions along the stem commercial height. According to the results obtained, it was possible to conclude that the end splitting on green and dry boards was influenced only by the diameter class, where the upper class presented the greatest length of splitting while the lower class showed the smallest length. A strong relationship between the end splitting of dry board and green board was verified.
\end{abstract}

Keywords: sawn wood, growth stresses, splitting.

\section{INTRODUÇÃO}

No Brasil, a indústria madeireira utiliza-se, em grande escala, de toras de árvores dos gêneros Pinus e Eucalyptus, com excelente aproveitamento de suas madeiras. Porém, espécies do gênero Liquidambar podem ser mais uma alternativa a ser usada pela indústria brasileira, principalmente na forma de madeira serrada. Algumas espécies desse gênero podem ter grande potencial para substituir as espécies 
que são mais utilizadas atualmente no Brasil. Entre as espécies do gênero Liquidambar, pode-se destacar a espécie Liquidambar styraciflua L., popularmente conhecida como "sweet gum"; esta espécie vem sendo muito utilizada e estudada, principalmente nos Estados Unidos, em função do seu grande potencial madeireiro e das suas características silviculturais (Shimizu \& Spir, 2004). Liquidambar styraciflua L. possui uma ampla distribuição geográfica natural, sendo encontrada em abundância nos Estados Unidos e no México, e em países da América Central, como Guatemala, Belize, El Salvador, Honduras e Nicarágua (Shimizu, 2005). A espécie possui grande potencial para reflorestamento pelo fuste retilíneo e pela boa qualidade de sua madeira, pois suas propriedades apresentam poucas variações, ao longo do tronco da árvore. Sua densidade especifica seria em torno de 0,52 g.m ${ }^{-3}$ (Carpenter \& Hopkins, 1966; Mattos et al., 2001).

L. styraciflua é utilizada há anos para fins medicinais, porém a atividade mais lucrativa para essa espécie, principalmente no sul dos Estados Unidos, é a produção de madeira serrada (Shimizu, 2005). Mattos et al. (2001) descrevem diferentes finalidades para a Liquidambar, como movelaria, caixaria, paletes, compensados, papel e polpa para celulose. Vários autores (Munoz, 1992; Gurgel Garrido, 1997; Mattos et al., 2001; Shimizu \& Spir, 2002) constataram a boa qualidade da madeira de Liquidambar, comparável à alcançada por algumas espécies dos gêneros Pinus e Eucalyptus. Mattos et al. (2001), estudando as características da madeira de L. styraciflua no Estado do Paraná, relatam a falta de informações em plantios experimentais com a espécie no Brasil, principalmente quanto às características da madeira. Os poucos estudos sobre a espécie informam que esta tem grande adaptação no sul e sudeste do País, e grande aproveitamento madeireiro. Contudo, novos estudos devem ser feitos sobre as formas de plantio, a qualidade da madeira e o melhoramento genético, por se tratar de uma espécie com grande potencial para programas desse tipo (Munoz, 1992).

Uma das principais razões dos baixos rendimentos encontrados na produção de madeira serrada, no Brasil é a ocorrência de rachaduras, principalmente para espécies folhosas. Esse defeito é originário da presença de elevadas tensões de crescimento nas árvores, antes da sua derrubada, atuando como uma forma de dar-lhes estabilidade para manter o equilíbrio (Van Wyk, 1978). De acordo com Opie et al. (1984), as causas das elevadas tensões de crescimento nas árvores não são bem conhecidas, mas há uma tendência de que estejam relacionadas a fatores genéticos, idades, dimensões da tora, taxa de crescimento e inclinação do fuste. Lisboa (1993) afirma que as tensões de crescimento têm efeitos benéficos para as árvores vivas, mas constituem graves dificuldades para o processamento da madeira, quando cortes transversais e longitudinais podem resultar em empenamentos e rachaduras. Segundo Hillis \& Brown (1984), a maioria das rachaduras de topo de tora deve ocorrer durante uma semana. Entretanto, rachaduras adicionais podem ocorrer durante o desdobro, quando há encurvamento longitudinal das peças, em razão das tensões residuais ao longo da tora. Essas rachaduras ou fendas podem ser intensas e estas distorções podem ser acentuadas durante a secagem. Portanto, as tensões de crescimento podem causar defeitos que forçam a redução das dimensões das peças obtidas, principalmente o comprimento (Kubler, 1987).

As tensões de crescimento existentes na madeira são definidas como forças que se desenvolvem no interior dos troncos das árvores vivas (Dinwoodie, 1966). Segundo Jacobs (1945), essas tensões são características intrínsecas ao crescimento natural das árvores e podem ocorrer tanto em folhosas como em coníferas. Segundo Ponce (1995), as tensões de crescimento não são exclusivas do gênero Eucalyptus, mas de todas as folhosas, sendo que esse autor já observou sinais evidentes de tensão de crescimento no mogno (Swietenia machrophylla), no jatobá (Hymenaea sp), na andiroba (Carapa guianensis), no cedro (Cedrela sp), na tatajuba (Bagassa guianensis) e na cupiúba (Goupia glabra); entretanto, algumas espécies apresentam tensões mais intensas do que outras, devendo-se, então, testar todas as espécies com potencial madeireiro.

De acordo com Varghese et al. (2000), a taxa de crescimento das árvores pode influenciar as propriedades das madeiras. Para Zobel (1981), a formação da madeira é um processo biológico que ocorre dentro da árvore viva, enquanto a qualidade da madeira é uma avaliação arbitrária de uma peça isolada de madeira, de uma parte da árvore ou de um derivado de madeira. Consequentemente, a qualidade da madeira somente pode ser alterada por 
meio do processo de formação dessa madeira. Assim, a competição entre árvores pode ser um fator a influenciar as propriedades das madeiras. Os fatores que podem afetar as propriedades da madeira são inerentes à própria madeira e ao ambiente em que a árvore se desenvolve (Latorraca \& Alburquerque, 2000).

O objetivo do presente trabalho foi estudar o comportamento das rachaduras durante o processo de secagem da madeira serrada de Liquidambar styraciflua, em função da posição da tora na árvore e das diferentes classes de diâmetro.

\section{MATERIAL E MÉTODOS}

As amostras de madeira foram coletadas de 12 árvores selecionadas em um plantio experimental de L. styraciflua com 24 anos, instalado na Estação Experimental de Mogi Mirim-SP, do Instituto Florestal, localizada nas coordenadas $22^{\circ} 26^{\prime} \mathrm{S}$ e $46^{\circ} 57^{\prime}$ W. O clima do local é do tipo Cwa, com precipitação anual de $1.355 \mathrm{~mm}$ e temperatura média anual de $20,3{ }^{\circ} \mathrm{C}$, com déficit hídrico anual de $19 \mathrm{~mm}$. O local apresenta uma altitude de $631 \mathrm{~m}$ e solo arenoso, sendo classificado como Latossolo Vermelho Amarelo, distrófico de textura média (Toledo Filho et al., 1989).

Inicialmente, realizou-se um inventário e, a partir da distribuição diamétrica, foram eleitas três classes de DAP (diâmetro à altura do peito; $1,30 \mathrm{~m}$ do solo) para o estudo, sendo a classe inferior (23 $\mathrm{cm})$, a classe mediana $(27 \mathrm{~cm})$ e a classe superior (33 $\mathrm{cm})$. Dentro de cada uma das classes, foram abatidas quatro árvores. Foram retiradas duas toras de cada uma das árvores selecionadas, sendo a primeira tora de 1,70 m de comprimento, retirada na base de cada árvore, e a segunda tora, subsequente, também de $1,70 \mathrm{~m}$. As toras selecionadas foram desdobradas em uma serra fita simples. De cada tora, foi retirada uma prancha central de $5 \mathrm{~cm}$ de espessura. A partir dessa prancha, determinaram-se os índices de rachaduras de extremidade de prancha nas condições verde e seca (ABNT, 1986).

\subsection{Indice de rachaduras de extremidade de prancha verde (IRPV)}

Esse índice representa a soma dos comprimentos das rachaduras das duas extremidades de cada uma das pranchas serradas na condição verde e foi calculado pela Equação 1:

$\operatorname{IRPV}=\left(C_{1}+C_{2}\right)$

em que: IRPV = índice de rachaduras de extremidade da prancha verde $(\mathrm{cm}) ; \mathrm{C}_{1}$ e $\mathrm{C}_{2}=$ comprimento das rachaduras respectivas das extremidades 1 e 2 da prancha na condição verde $(\mathrm{cm})$.

\subsection{Indice de rachaduras de extremidade de prancha seca (IRPS)}

As pranchas na condição verde passaram por um processo de secagem ao ar livre, por um período de quatro meses. A cada 30 dias, foi avaliado o comprimento das rachaduras até a condição seca para se obter o índice de rachaduras de extremidade de prancha seca (IRPS), que representa a soma dos comprimentos acumulados das rachaduras das duas extremidades de cada uma das pranchas no estado seco, calculado pela Equação 2:

IRPS $=\left(C_{1}+C_{2}\right)$

em que: IRPS = índice de rachaduras de extremidade de prancha seca $(\mathrm{cm}) ; \mathrm{C}_{1}$ e $\mathrm{C}_{2}=$ comprimento das rachaduras respectivas das extremidades 1 e 2 da prancha na condição seca $(\mathrm{cm})$.

As hipóteses testadas neste trabalho foram: a) A classe diamétrica não influencia nos índices de rachaduras nos estados verde e seco das pranchas de madeira de L. styraciflua; b) Não existem variações nos índices de rachaduras nos estados verde e seco das pranchas de madeira de L. styraciflua ao longo da altura das toras.

$\mathrm{Na}$ avaliação do experimento, foi efetuado inicialmente $o$ teste de homogeneidade de variância e, para isso, utilizou-se o teste de Hartley. Posteriormente, aplicou-se o teste $\mathrm{F}$ de análise de variância segundo o delineamento experimental inteiramente casualizado, adotando-se o esquema fatorial $3 \times 2$ (classe $\times$ tora). Aplicou-se o teste de Tukey sempre que observada diferença significativa, no nível de 5\% de significância, entre os tratamentos. Um estudo de relação entre IRPV e IRPS também foi realizado e, para isso, utilizou-se de análise de regressão, em nível de significância de 5\%. Os dados obtidos das variáveis foram analisados estatisticamente com o auxílio do procedimento estatístico PROC GLM e PROC REG (SAS, 1999). 


\section{RESULTADOS E DISCUSSÃO}

$\mathrm{Na}$ Tabela 1, encontra-se o resumo das análises de variância realizadas para as variáveis IRPV e IRPS.

O IRPV e o IRPS diferiram significativamente, entre as classes de DAP (Tabela 1). Isso demonstrou que os diâmetros das árvores influenciaram significativamente os índices de rachaduras, sendo que a classe superior apresentou maior intensidade de rachadura e a classe inferior, o menor índice de rachadura (Figuras 1 e 2).

Essa diferença entre os níveis de significância das classes superior e inferior pode ser explicada pela relação entre o comprimento da rachadura e a largura da prancha ter sido menor na classe inferior, e maior na classe superior. Outra explicação, segundo Kubler (1987), pode ser o fato de as árvores da classe inferior terem crescido mais lentamente e, assim, ter-

Tabela 1. Resumo de análise de variância efetuada para os índices de rachaduras de extremidade de prancha verde (IRPV) e seca (IRPS) de L. styraciflua de 24 anos de idade

Table 1. Summary of analysis of variance for green board end splitting index (IRPV) and dry board end splitting index (IRPS) in 24-year old grown L. styraciflua.

\begin{tabular}{lccc} 
Causa de variação & GL & \multicolumn{2}{c}{ Quadrados médios } \\
\cline { 3 - 4 } & & IRPV $(\mathbf{c m})$ & IRPS $(\mathbf{c m})$ \\
\hline Classe & 2 & $265^{* *}$ & $577^{* *}$ \\
Tora & 1 & $74^{\text {n.s. }}$ & $64^{\text {n.s. }}$ \\
\hline Classe $\times$ Tora & 2 & $47^{\text {n.s. }}$ & $62^{\text {n.s. }}$ \\
Resíduo & 18 & 51 & 67 \\
Média & & 5,76 & 16,01 \\
\hline
\end{tabular}

${ }^{* *}$ significativo no nível de $1 \%$ de significância; ${ }^{n . s}$ não significativo no nível de $5 \%$ de significância.

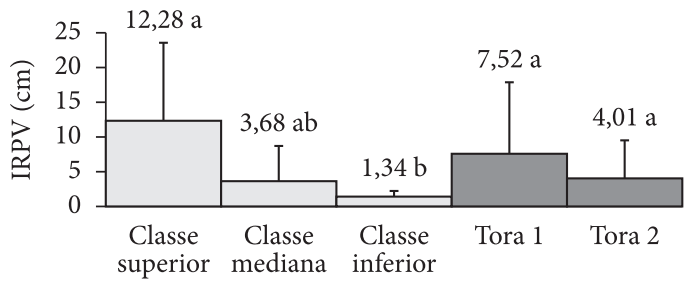

Figura 1. Índice de rachaduras de extremidade de prancha verde (IRPV) de L. styraciflua de 24 anos de idade. Médias com a mesma letra não diferem entre si pelo teste Tukey a $5 \%$ de significância.

Figure 1. Green board end splitting index (IRPV) in 24year old grown L. styraciflua. se-ia acumulado menos tensão no tronco. A mesma tendência foi verificada por Crêspo (2000), que encontrou em populações de E. grandis e E. saligna valores maiores de rachaduras de extremidade de tábua na classe superior e, a partir disso, concluiu que tal fato ocorreu pela tendência de a classe superior produzir tábuas de larguras maiores do que aquelas obtidas de classes inferiores. Lima et al. (2006) também verificaram que tábuas de E. grandis, em estado verde, oriunda das árvores da classe superior, apresentaram maior intensidade de rachadura do que as das classes mediana e inferior. Lima \& Garcia (2008) verificaram, para uma população de E. grandis, que os índices de rachaduras de tábuas verdes e secas foram influenciados significativamente pelas diferentes classes de DAP.

Trevisan et al. (2009) observaram que árvores dominantes de E. grandis, que cresceram em maior espaço vital, apresentam menor índice de rachaduras de tábuas, enquanto que as árvores da classe mediana não apresentaram essa mesma tendência.

Malan \& Hoon (1992) e Lima et al. (2000) não verificaram efeitos significativos de diferentes intensidades de desbastes no comprimento de rachaduras de extremidade de tábua, em diferentes classes de DAP, em uma população de E. grandis. Entretanto, Miranda e Nahuz (1999) observaram que espaçamentos maiores entre árvores provocaram menores índices de rachaduras das tábuas, após a secagem. Porém, de acordo com Fernandes (1982), Malan \& Hoon (1992) e Lopes et al. (2004), existe um alto controle genético nas rachaduras de extremidade de tábua, tendo, então, o fator ambiental pouca influência sobre a intensidade das rachaduras.

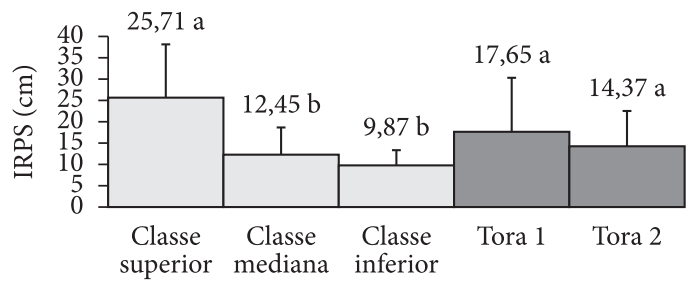

Figura 2. Índice de rachaduras de extremidade na prancha central seca (IRPS) de L. styraciflua de 24 anos de idade. Médias com a mesma letra não diferem entre si pelo teste Tukey a $5 \%$ de significância.

Figure 2. Dry board end splitting index (IRPV) in 24year old grown L. styraciflua. 
Contudo, Ferrand (1983), em um trabalho com E. delegatensis e E. nitens, verificou que desbastes moderados não influenciaram na deformação de crescimento, mas desbastes precoces e pesados influenciaram significativamente e, segundo o autor, isso ocorreu porque a deformação de crescimento é fortemente relacionada com a competição entre árvores.

Trugilho et al. (2007) observaram baixas correlações entre a DRL (Deformação Residual Longitudinal), que é uma medida indireta da tensão de crescimento, e as características de crescimento; segundo esses autores, este resultado indica que a tensão de crescimento também não deve ser afetada pelas características de crescimento. Valencia et al. (2011) também verificaram em uma população de $E$. nitens, de 22 anos, que a DRL não tem relação com o diâmetro das árvores. Entretanto, Carvalho et al. (2010) verificaram que a DRL tem correlação com as classes de DAP, sendo que as mesmas mostraram-se negativas e significativas; dessarte, para esses autores, a classe de DAP é um parâmetro a ser utilizado em seleções de indivíduos, com menores intensidades de tensões de crescimento tanto em populações de Corymbia citriodora como de E. urophylla.

Em relação à posição da tora na altura da árvore, apesar de as toras da base apresentarem maiores IRPV e IRPS, não ocorreram diferenças significativas entre as posições das toras (Tabela 1 e Figuras $1 \mathrm{e}$ 2). Isso pode ser explicado pelo fato de as toras 1 e 2 apresentarem baixa conicidade, ou seja, as dimensões da largura das pranchas das toras 1 e 2 são muito próximas; dessa forma, considerando-se que as rachaduras têm alta relação com as larguras das tábuas, era de se esperar, então, que não ocorressem diferenças entre as toras 1 e 2 . O fato de as rachaduras serem dependentes das larguras das tábuas foi verificado por Lima et al. (2000) e Lima \& Garcia (2008).

Pode-se verificar, por meio do teste F, que a interação 'classe diamétrica $\times$ posição vertical' não foi significativa, indicando então não existir uma dependência entre os efeitos dos fatores, o que mostra que o padrão de variação de IRPV e IRPS, tora 1 e tora 2 , é o mesmo para todos os tratamentos (Tabela 1).
Na Figura 3, há uma representação da evolução da rachadura do estado verde para o seco, durante o período de secagem. Foi verificado que o modelo de regressão logarítmico foi significativo para essa relação, sendo possível estimar o comprimento de rachadura propagado durante a secagem da madeira (Figura 3). Verifica-se também que ocorreu uma tendência de um aumento do valor da rachadura ao longo do tempo. Comparando-se o valor médio de IRPV $(5,76 \mathrm{~cm})$ com o valor médio de IRPS $(16,01 \mathrm{~cm})$, verifica-se que houve uma propagação da rachadura, que existia na prancha verde durante o processo de secagem, e que esse aumento médio foi de 10,25 cm (Tabela 1). De acordo com Kubler (1987), isso é explicado pelo fato de a maioria das rachaduras ocorrer durante os primeiros dias após a derrubada da árvore. Entretanto, rachaduras adicionais devem ocorrer durante o desdobro, quando há encurvamento longitudinal das peças em virtude das tensões residuais ao longo da tora. Essas rachaduras podem ser intensas e se acentuarem durante o período de secagem. Isto normalmente ocorre em algumas espécies do gênero Eucalyptus de crescimento rápido. De acordo com Garcia (1997), essa perda poderia ser amenizada evitandose trabalhar com toras de pequenos comprimentos, já que cada corte transversal efetuado no tronco da árvore produzirá duas extremidades livres e, portanto, sujeitas às rachaduras. Lima \& Garcia (2008) verificaram em tábuas de E. grandis um aumento de $10,82 \mathrm{~cm}$ nas rachaduras durante o período de secagem, ou seja, 120 dias.

De acordo com a análise da relação entre IRPV e IRPS, em que foi testado o modelo de regressão linear, verificou-se uma forte dependência entre rachaduras

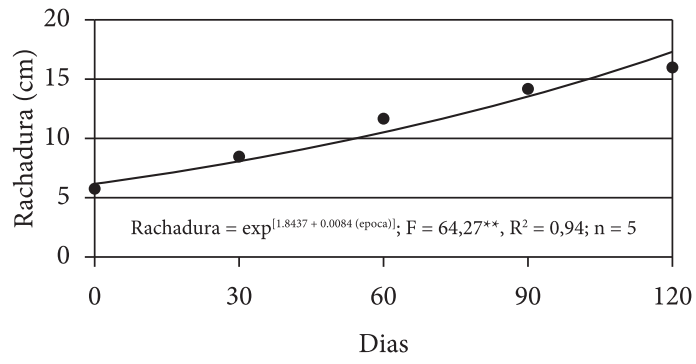

Figura 3. Rachadura na prancha central de L. styracifluant em função do tempo de secagem.

Figure 3. The index end splitting of board in L. styracifluant as affected by drying period. 


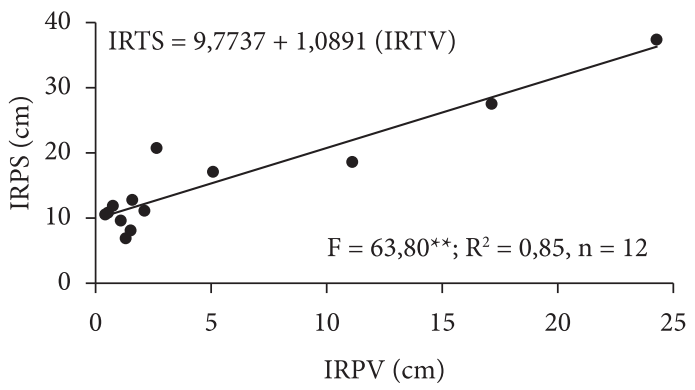

Figura 4. Índice de rachaduras de extremidade na prancha central seca (IRPS) e Índice de rachaduras de extremidade de prancha verde (IRPV).

Figure 4. Dry board end splitting index (IRPS) and green board end splitting index (IRPV).

de pranchas seca e verde, o que permitiu estimar, com certa segurança, a perda de comprimento que a prancha terá durante o processo de secagem (Figura 4).

Lima et al. (2000), Malan (2000) e Lima \& Garcia (2008) também estudaram essa relação em tábuas de E. grandis e obtiveram resultados semelhantes entre essas variáveis.

De acordo com o exposto, verifica-se que a madeira de L. styraciflua apresenta os efeitos da tensão de crescimento e secagem, na forma de rachaduras, porém com uma tendência de tais efeitos serem menos acentuados do que é observado principalmente em algumas espécies do gênero Eucalyptus.

\section{CONCLUSÕES}

Os índices de rachaduras de pranchas verdes e secas foram influenciados significativamente pelas diferentes classes de DAP, rejeitando-se assim a hipótese de que o diâmetro das árvores não influencia nos índices de rachaduras. As árvores de maior diâmetro apresentam maiores intensidades de rachaduras e as de menor diâmetro, menores intensidades de rachaduras.

Os índices de rachaduras de pranchas verdes e secas não foram influenciados significativamente pelas posições das toras, na altura da árvore, aceitando-se assim a hipótese de não haver variações significativas ao longo da altura das arvores.

A estimativa do comprimento de rachadura propagado durante o processo de secagem foi muito boa.
Houve uma relação positiva entre rachaduras de prancha verde e rachaduras de prancha seca.

\section{STATUS DA SUBMISSÃO}

Recebido: $18 / 10 / 2011$

Aceito: 26/11/2012

Publicado: 28/02/2013

\section{AUTOR(ES) PARA CORRESPONDÊNCIA}

\section{Israel Luiz de Lima}

Florestal do Estado de São Paulo,

Divisão de Dasonomia, Rua do Horto, 931,

CEP 02377-000, São Paulo, SP, Brasil

e-mail: israelluizde.lima@yahoo.com.br

\section{REFERENNCIAS}

Associação Brasileira de Normas Técnicas - ABNT. NBR 94987: Classificação de madeira serrada de folhosas. São Paulo: ANBT; 1986. 53 p.

Carpenter BE, Hopkins WC. Specific gravity values of sweetgum (Liquidambar styraciflua) topwood and bolewood. Forest Products Journal 1966; 6(7): 30.

Carvalho AM, Gonçalves MPM, Amparado KF, Latorraca JVF, Garcia RA. Correlações da altura e diâmetro com tensões de crescimento em árvores de Corymbia citriodora e Eucalyptus urophylla. Revista Árvore 2010; 34(2): 323-331. http://dx.doi.org/10.1590/ S0100-67622010000200015

Crêspo EA. Tensão de crescimento e suas conseqüências, controláveis e não controláveis, no desdobro e secagem do Eucalyptus grandis e E. saligna Muell. [dissertação] Piracicaba: Escola Superior de Agricultura "Luiz de Queiroz”, Universidade de São Paulo; 2000.

Dinwoodie JM. Growth stresses in timber - a review of literature. Forestry 1966; 39(2): 162-170. http://dx.doi. org/10.1093/forestry/39.2.162

Fernandes PS. Variações de densidade da madeira e suas relações com as tensões de crescimento em progênies de Eucalyptus urophylla S. T. Blake [dissertação]. Piracicaba: Escola Superior de Agricultura "Luiz de Queiroz", Universidade de São Paulo; 1982.

Ferrand JC. Growth Stresses and Silviculture of Eucalyptus. Australian Forest Research 1983; 13: 175-81.

Garcia JN. An alternative Sawmill Plant To Improve Eucalyptus Lumber Quality. In: Proceedings of the International Wood Machining Seminar, 1997, Vancouver. Vancouver; 1997. p. 17-20. 
Gurgel Garrido LMA, Faria HH, Cruz SF, Palomo M. Variabilidade genética de característica silviculturais de liquidambar (Liquidambar styraciflua L.) em teste de origens em Paraguaçu Paulista - SP. Revista do Instituto Florestal 1997; 9(2): 125-132.

Hillis WH, Brown AG. Eucalypts for wood production. Melbourne: CSIRO; 1984. 434 p.

Jacobs, MR. The growth of woody stems. Bulletin Commonwealth Forests Bureau 1945; 28:67.

Kubler H. Growth Stresses in Trees and Related Wood Properties. Forestry Abstracts, 1987; (48): 131-189.

Latorraca JVF, Albuquerque CEC. Efeito do rápido crescimento sobre as propriedades da madeira. Floresta e Ambiente 2000; 7(1): 279-91.

Lima IL, Garcia JN. Influência do desbastee da fertilização nos índices de rachamento após desdobro e secagem em madeira serrada de Eucalyptus grandis Hill ex-Maiden. Revista do Instituto Florestal 2008: 20(2):175-184.

Lima IL, Garcia JN, Stape JL, Piedade SMS. Efeito do desbaste e da fertilização nas tensões de crescimento em Eucalyptus grandis. Scientia Forestalis 2006; 70: 171183.

Lima IL, Garcia JN, Nogueira MCS. Influência do desbaste nas tensões de crescimento de E. grandis Hill ex-Maiden. Scientia Forestalis 2000; 58: 111-125.

Lisboa CDJ. Estudo das tensões de crescimento em toras de Eucalyptus grandis Hill ex Maiden [tese]. Curibitiba: Universidade Federal do Paraná; 1993.

Lopes MC, Haselein CR, Santini EJ, Longhi SJ, Rosso $\mathrm{S}$, Fernandes DLG et al. Agrupamentos de árvores matrizes de Eucalyptus grandis em função das variáveis dendrométricas e das caracterização tecnológicas da madeira. Ciência Florestal 2004; 14(2): 133-144.

Malan FS, Hoon M. Effect of initial spacing and thinning on some wood properties of Eucalyptus grandis. South African Forestry Journal, 1992; 163: 13-20. http://dx.doi. org/10.1080/00382167.1992.9629362

Malan FS. The wood properties and sawn board quality of the Eucalyptus grandis x E. urophylla hybrid. South African Forestry Journal 2000; 188: 29-35. http://dx.doi. org/10.1080/10295925.2000.9631267

Mattos PP, Pereira JCD, Schaitza EG, Carvalho PER. Características da madeira de Liquidambar styraciflua. Circular Técnica Embrapa Florestas 2001; 49: 1-4.

Miranda MJAC, Nahuz MAR. Estudo da influência do espaçamento de plantio de Eucalyptus saligna Smith nos índices de rachamento após o desdobro e após a secagem. Scientia Forestalis 1999; 55: 107-116.

Munoz VL. Apuntes sobre algunas latifoliadas de maderas valiosas: liquidambar (Liquidambar styraciflua
L.). Ciencia e Investigación Forestal 1992; 6(2): 335 48, 1992.

Opie JE, Curtin RA, Incoll WD. Stand management. In: Hillis W, Brown AG. Eucaliptos for wood production. Melborne: CSIRO; 1984. 434 p.

Ponce RH. Madeira Serrada de Eucalipto: desafios e perspectivas. In: Seminário Internacional de Utilização da Madeira de Eucalipto para Serraria; 1995; São Paulo. São Paulo; 1995. p. 50-8.

Shimizu JY. Liquidambar para produção de madeira no Sul e Sudeste do Brasil. Boletim de Pesquisa Florestal 2005; 50:127-138.

Shimizu JY, Spir IHZ. Produtividade de madeira de liquidambar (Liquidambar styraciflua L.) de diferentes procedências, em Quedas do Iguaçu, PR. Revista Árvore 2004; 28(4): 487-491. http://dx.doi.org/10.1590/ S0100-67622004000400003

Shimizu JY, Spir IHZ. Produtividade de madeira de liquidambar de diferentes procedências em Quedas do Iguaçu, PR. Boletim de Pesquisa Florestal 2002; 44: 3-12.

Statistical Analisys System Institute - SAS. SAS Procedures Guide. version 8 (TSMO). Cary: SAS Institute Inc.; 1999.

Toledo Filho DV, Leitão Filho HF, Shepherd GJ. Estrutura fitossociológica da vegetação do cerrado em Moji-Mirim (SP). Revista do Instituto Florestal 1989; 1(2): 1-12.

Trevisan R, Haselein CR, Santini EJ, Schneider PR, Menezes LF. Efeito da intensidade de desbaste na qualidade da madeira serrada de Eucalyptus grandis. Floresta 2009; 39(40): 825-831.

Trugilho PF, Rosado SCS, Lima JT, Pádua FA, Souza MAM. Deformação residual longitudinal (DRL) e sua relação com as características de crescimento da árvore em clones de Eucalyptus. Cerne 2007; 13(2): 130-137.

Valencia J, Harwood C, Washusen R, Morrow A, Wood M, Volker P. Longitudinal growth strain as a $\log$ and wood quality predictor for plantationgrown Eucalyptus nitens sawlogs. Wood Science and Technology 2011; 45: 15-34. http://dx.doi.org/10.1007/ s00226-010-0302-1

Van Wyk JL. Hardwood sawmilling can have a bright future in South Africa. South African Forestry Journal 1978; 107: 47-53. http://dx.doi.org/10.1080/207 02620.1978 .10433505

Varghese M, Nicodemus A, Ramteke PK, Anbazhagi G, Bennet SSR, Subramanian K. Variation in Growth and Wood Traits Among Nine Populations of Teak in Peninsular Índia. Silvae Genetica 2000; 49(2-5): 201 205.

Zobel B. Wood quality from fast-grown plantations. Tappi 1981; 64(1): 17-74. 